\title{
Contributing Factors to Complications and Surgical Success in Mouse Kidney Transplantation
}

\author{
Ling-Jin Huang, Shannon Reese, Arjang Djamali
}

Department of Medicine, University of Wisconsin Madison SMPH, (LJH, SR, AD), Madison, WI; Department of Surgery, University of Wisconsin Madison SMPH, (LJH, AD), Madison, WI, USA and Department of Cardiothoracic Surgery, Xiangya Hospital, Central South University (LJH), Changsha, Hunan, PRC

\section{ABSTRACT}

Purpose: Mouse kidney transplantation is a challenging technique for novice microsurgeons. Factors that affect transplant outcomes for a clinical surgeon starting microsurgery have not yet been investigated.

Materials and Methods: 110 consecutive mouse kidney transplants were performed over a 9-month period. Data were recorded, and surgical results and complication were analyzed.

Results: Three and thirty day survival rates improved from $0(0 / 6)$ to $92.3 \%$ (12/13) between months 1 and 9. Bleeding, arterial thrombosis, kidney failure and hydronephrosis were the most common causes of transplant failure. From month 1 to month 7 , using the same surgical technique, practice significantly decreased the incidence of bleeding and increased the 3-day survival rate; however, it didn't significantly decrease the incidence of thrombosis, kidney failure, but improved the 30-day survival rate. From month 8, when surgical technique used on artery anastomosis switched from continuous suture to interrupted suture, surgical survival rate at 3 and 30 days improved significantly. Interestingly, ischemia time was not a significant factor determining the success of transplantation in this study.

Conclusions: Practice is essential for novice microsurgeons, and the choice of surgical techniques significantly affects surgical results. The use of interrupted arterial sutures can significantly improve mouse kidney transplantation outcomes compared with continuous sutures. Ischemic time was not a factor in determining successful of kidney transplantation in mice in this study.

\section{ARTICLE INFO}

\section{Key words:}

Kidney transplantation; mice; urologic surgical procedures; male; survival rate

Int Braz J Urol. 2012; 38: 395-404

Submitted for publication:

December 21, 2011

Accepted after revision:

March 16, 2011

\section{INTRODUCTION}

Mouse kidney transplantation is an ideal model for transplant research because genetically modified strains can be used to study the molecular mechanisms of renal allograft injury $(1,2)$. However, due to the technical complexity and high mortality rates, only a handful transplant centers uses this model for research. The first mouse kidney transplantation was described by Skoskiewicz et al. in 1973 (3), later improved by Zhang and Han $(4,5)$. To become familiar with the technique, reduce complications and increase survival rate, a relatively long learning curve is needed even for experienced microsurgeons (6). 
Although there are papers describing the procedure of mouse kidney transplantation in detail, the factors that contribute to successful transplantation have not yet been evidencedbased investigated (7). In this report, a surgeon with 10 years of experience in cardiothoracic surgery started his microsurgery training on mouse. Data from the first 110 mouse kidney transplants were collected to investigate the factors contributing to successful mouse kidney transplantation. An understanding of these factors will be helpful for future researchers who plan to use this model.

\section{MATERIALS AND METHODS}

\section{Animals}

Male C57BL/6 and BALB/c mice, 6-8 weeks old and weighing between 18 and $25 \mathrm{~g}$, were used for kidney transplantations. Mice were bred and maintained in the animal care facility at the William Middleton VA Hospital in Madison, Wisconsin. Procedures were performed in accordance with the Animal Care Policies at the VA Hospital and the University of Wisconsin. In total, 110 mouse kidney transplantations were performed between February and October of 2010. Surgical times, complications and results of each of the surgeries were recorded and analyzed.

\section{Anesthesia and surgical techniques}

Mice were anesthetized with Isoflurane (2-3\%). The transplants were performed using the technique described by Zhang and Han $(4,5)$. All procedures were performed at 4-25X magnification using a microscope (SMZ800, Nikon, Japan) and standard microsurgical instruments.

Briefly, a midline abdomen incision was performed. The left kidney was exposed by moving the intestine, spleen and stomach to the right side. The suprarenal and infrarenal aorta and inferior vena cava (IVC) were dissected with ligation and division of the attached lumbar branches. The aorta and IVC were then dissected apart below the renal pedicle for a length of 3-4 $\mathrm{mm}$. The adrenal and testicular vessels were divided and ligated with 10-0 nylon sutures (AROSurgical, CA, USA). These ties could be used to orient the graft during the procedure of transplantation. The kidney was then separated from perinephric fat and tissues. The left ureter was dissected free down to the bladder preserving the periureteral vessels and cut with a small bladder patch. Using an 8-0 nylon suture (AROSurgical, CA, USA), the infrarenal aorta and IVC were ligated, and the same procedure was done to the suprarenal aorta and IVC. The IVC was then transected below the renal vein. The donor kidney was then perfused via the infrarenal aorta with $1 \mathrm{~mL}$ cold heparinized normal saline $(100 \mathrm{U} / \mathrm{mL})$. The aorta was cut approximately $2 \mathrm{~mm}$ below the renal artery. The kidney and its vascular supply was harvested with the ureter attached to the bladder patch en bloc, and then stored in $4^{\circ} \mathrm{C}$ normal saline until the time of transplantation.

A two-stage procedure was performed in the recipients. First, the left native kidney was removed and the donor kidney was transplanted into the recipient. Second, the contralateral native kidney was removed on post-transplantation day 10 . Briefly, with a midline abdomen incision, left native kidney was removed after the vascular pedicle and ureter were ligated. The infrarenal aorta and IVC were isolated carefully and cross clamped using two 8-0 suture releasable knots (Figure-1A). The use of releasable knot can provide more room in the surgical field, and less mobilization and dissection is needed. Other benefits included less cost and very easy to tie the knot and release it (Figure-1B). Small elliptical aortotomy and venotomy were made. Endto-side vascular anastomoses were performed. A running 11-0 nylon suture (AROSurgical, CA, USA) was used in the first continuous 86 transplantation surgeries from month 1 to 7 . The last 24 surgeries at month 8 and 9 used interrupted suture for the artery anastomosis. Donor vein and recipient IVC was connected using continuous 11-0 sutures. Once the venous anastomosis was completed, the releasable knots were released and the kidney graft was perfused instantly when the anastomosis was successful.

Urinary reconstruction was established with ureteral implantation technique with some modification (5). Briefly, the recipient bladder was pierced with a fine tweezers (Dumont Twee- 
Figure 1 - A, The infrarenal aorta and IVC were cross clamped using two 8-0 suture releasable knot instead of micro clamps. B, Sketch of releasable knot. Pull end 1 and 2 tighten the knot, pull end 3 release the knot.

A

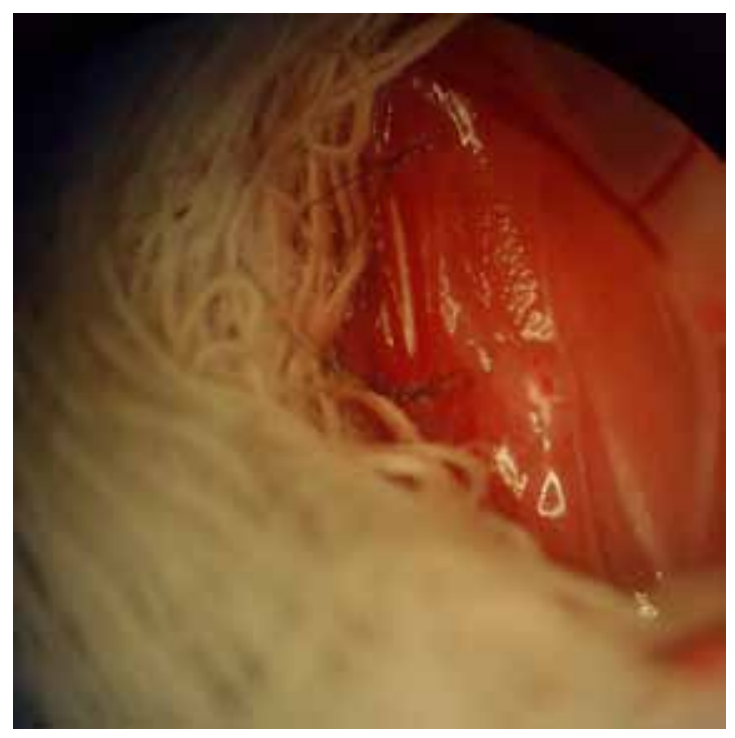

zers \#5, WPI Co. USA) and the donor ureter was pulled through the bladder. Periureteral tissue was fixed to the exterior wall of the bladder using 10-0 sutures. 2-3 $\mathrm{mm}$ length of the donor ureter was left and allowed to retract inside the bladder before closing the contralateral pierced wound.

Transplanted mice were monitored daily. Three and thirty-day recipient survival were counted and analyzed. According to the proposal, kidneys, urine and blood of thirty-day survival recipient were harvested for further examination. Following the harvest, mice were euthanized by exsanguination.

\section{Statistical Analysis}

Multivariable analysis was used to examine the effects of the various variables including practice (month), bleeding, thrombosis and kidney failure on surgical survival. Surgical times are presented as mean \pm standard deviation, and statistical significance was assessed using unpaired t test. Fisher's exact test was used for the

\section{B}

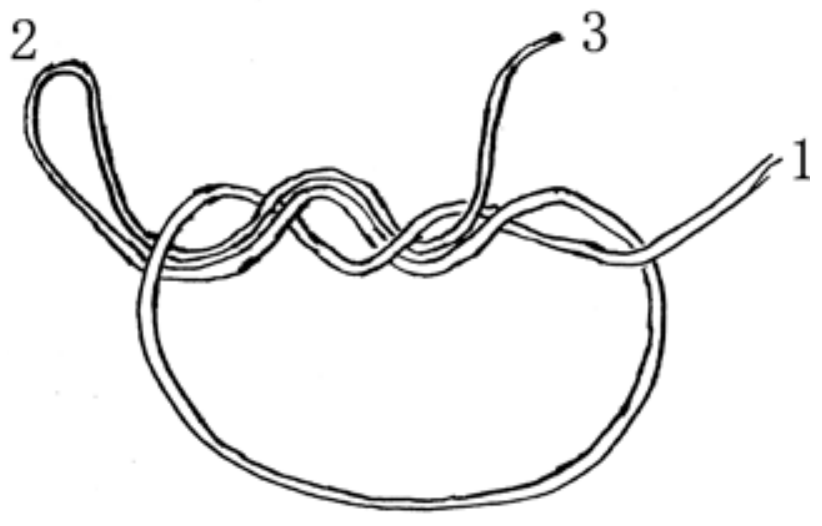

comparison of different anastomosis method in artery. All data analyses were conducted using SPSS v 17.0 (SPSS, Chicago IL), and P $<0.05$ was considered statistically significant.

\section{RESULTS}

Surgical results and complications

One hundred and ten consecutive kidney transplants were performed between February and October of 2010. Three and thirty day's survival rate improved from 0 to $92.3 \%$, and 0 to 92.3\% respectively (Table-1).

Causes of death or euthanasia included bleeding, thrombosis, kidney failure, urine leakage, vena cava complication, and hydronephrosis. Bleeding, thrombosis and kidney failure accounted for over 70\% of the complications in the first 3 months of training (Table-1).

Bleeding was the cause of nearly all deaths in the first two months of training. With the improvement in microsurgical technique and use of Surgicel ${ }^{\circledR}$, the incidence of bleeding decreased rapidly after the 3rd month, accounting 
Table 1 - Surgical results by month.

\begin{tabular}{lcccccc}
\hline Month & Surgery & Bleeding (rate) & $\begin{array}{c}\text { Thrombosis } \\
\text { (rate) }\end{array}$ & $\begin{array}{c}\text { Kidney failure } \\
\text { (rate) }\end{array}$ & $\begin{array}{c}\text { 3-day survival } \\
\text { (rate) }\end{array}$ & $\begin{array}{c}30 \text {-day survival } \\
\text { (rate) }\end{array}$ \\
\hline 1 & 6 & $5(83.3 \%)$ & $1(16.7 \%)$ & - & 0 & 0 \\
2 & 16 & $10(62.5 \%)$ & $3(19.0 \%)$ & - & $3(19.0 \%)$ & 0 \\
3 & 15 & $3(20.0 \%)$ & $7(46.7 \%)$ & - & $4(26.7 \%)$ & $3(20.0 \%)$ \\
4 & 16 & $2(12.5 \%)$ & $6(37.5 \%)$ & $1(6.3 \%)$ & $8(50.0 \%)$ & $4(25.0 \%)$ \\
5 & 15 & $2(13.3 \%)$ & $1(6.7 \%)$ & $4(26.7 \%)$ & $12(80.0 \%)$ & $2(13.3 \%)$ \\
6 & 8 & $2(25.0 \%)$ & $3(37.5 \%)$ & 0 & $3(37.5 \%)$ & $2(25.0 \%)$ \\
7 & 10 & 0 & $3(30.0 \%)$ & $6(60.0 \%)$ & $7(70.0 \%)$ & $1(10.0 \%)$ \\
8 & 11 & $1(9.1 \%)$ & $1(9.1 \%)$ & $1(9.1 \%)$ & $8(72.7 \%)^{*}$ & $7(63.6 \%)^{*}$ \\
9 & 13 & $1(7.7 \%)$ & 0 & 0 & $12(92.3 \%)$ & $12(92.3 \%)$ \\
\hline
\end{tabular}

From month 1 to month 7 , the same surgical technique was used, there was significant effect of practice on bleeding $(P=0.017)$ and 3-day survival $(P=0.025)$, but no significant difference was found of practice on thrombosis and kidney failure $(P=0.767$ and 0.080 respectively). From month 8 , artery anastomosis switched from running suture to interrupted suture. 3 - and 30 -day survival rate increased significantly ( ${ }^{*}$ Compared with month $7, \mathrm{P}<0.05$ ).

for only 10 to $20 \%$ of all subsequent deaths. The second most common complication was thrombosis, which remained a significant obstacle until month 8, when a switch to interrupted sutures on artery anastomosis improved the results. The third more prevalent complication was kidney failure. In these mice, transplantations were successful when grafts perfused very well, and the graft was pink and the renal artery patency could be observed on the second stage surgery (bilateral nephrectomy), which indicated that graft perfusion existed. However, the mice died 2 or 3 days later after the second stage surgery. In month 7, 60\% mice died due to this complication. There were five cases of hydronephrosis in all surviving mice, and urine leak caused serious sickness in two mice.

\section{Effects of practice on complications}

Surgical results are always dependent on practice. In these serial surgeries, different months represented different proficiency of sur- gical practice. Because of the use of the same surgical procedure from month 1 to month 7 , data were analyzed to determine whether practice (different month) could decrease the incidence of different complications. As indicated in Table-1, there was a significant effect of practice on bleeding $(\mathrm{P}=0.017)$. However, no significant difference was found of practice on thrombosis or kidney failure $(\mathrm{P}=0.767$ and 0.080 respectively).

Association between practice (month) and surgical survival

Since survival is the most important endpoint in microsurgery, the data was investigated further to determine the association between the practice (month) and the survival rate. From month 1 to month 7, the same surgical technique was used (continuous artery anastomosis). The effect of practice on surgical survival rates was analyzed. Findings showed that practice improved the 3 -day survival rate $(\mathrm{P}=0.025)$. How- 
ever, there was no better change in the elevation of 30-day survival rate $(\mathrm{P}=0.221)$, which indicated that other factors may be involved in the improvement of the long-term survival rate.

Difference between using continuous and interrupted suture on artery anastomosis

From month 8, interrupted artery suture method was used. Obvious difference was found at 30-day survival rate. Data of month 7 and month 8 which used continuous and interrupted suture in artery anastomosis was analyzed separately. The use of interrupted suture method resulted in a higher 30-day survival rate than using the continuous suture method; this difference was probably due to a lower incidence of kidney failure in the interrupted suture method group (Table-2). Significant difference was also found in 3-day survival rate between these two methods
CIT) and warm ischemia time (WIT). CIT was the time duration of donor kidney preserved in cold saline. WIT was the time duration to complete the vessel anastomosis between donor kidney and recipient, out from the cold saline. Practice could decrease the ischemia times gradually (Figure-2B), and there was significantly difference $(\mathrm{P}<0.05)$.

\section{Impact of ischemia time on the results}

One of the most important factors impacting transplantation results is ischemia time. When the continuous artery anastomosis technique was used (from month 1 to month 7), the ischemia time differed among the groups of 30-day survival, thrombosis and kidney failure. It seemed as if there was a relationship among the groups, but there were no significant differences. Ischemia time is important, but it was not associated with surgical survival in this procedure (Table-3).

Table 2 - Surgical results of different method of artery anastomosis.

\begin{tabular}{llllllll}
\hline Method & Number & $\begin{array}{l}\text { Bleeding } \\
\text { (rate) }\end{array}$ & $\begin{array}{l}\text { Thrombosis } \\
\text { (rate) }\end{array}$ & $\begin{array}{l}\text { Kidney failure } \\
\text { (rate) }\end{array}$ & WIT & $\begin{array}{l}\text { 3-day survi- } \\
\text { val (rate) }\end{array}$ & $\begin{array}{l}\text { 30-day sur- } \\
\text { vival (rate) }\end{array}$ \\
\hline $\begin{array}{l}\text { Continuous suture } \\
\text { Interrupted }\end{array}$ & 10 & 0 & $3(30.0 \%)$ & $6(60.0 \%)$ & $29.58 \pm 5.12$ & $7(70.0 \%)$ & $1(10.0 \%)$ \\
\begin{tabular}{l} 
suture \\
\hline
\end{tabular} & 11 & $1(9.1 \%)$ & $1(9.1 \%)^{*}$ & $1(9.1 \%)^{*}$ & $31.67 \pm 6.12$ & $8(72.7 \%)^{\star}$ & $7(63.6 \%)^{\star}$ \\
\hline
\end{tabular}

Kidney transplantation data using interrupted suture in Month 8 was compared with the use of continuous suture in Month 7. Interrupted suture method significantly decreased the incidence of thrombosis and kidney failure, and significantly improved surgical survival rate. There was no significant difference on warm ischemia time $(p=0.398) .{ }^{*}: P<0.05$, Compared with continuous suture group. WIT: Warm ischemia time. Time duration on vessel anastomosis (out of cold saline preservation).

$(P=0.012)$. There was no significant difference in the warm ischemia time between these two methods (Table-2).

\section{Surgical Time}

With practice, surgical time on donor and recipient reduced to 50 minutes and 100 minutes respectively (Figure-2A). The total ischemia time (TIT) in the transplantation consisted of two parts: cold saline preservation time (Cold ischemia time,

\section{DISCUSSION}

The mouse kidney transplantation model is challenging for microsurgeons and has a steep learning curve. In addition to technical requirements, the beginner should also understand variable factors including ischemia time, complications and suturing methods which are related to surgical outcomes. Here we described our experience in 110 consecutive mouse kidney transplants. 
Figure $2 \mathrm{~A}$ and B - (A) Surgical time in mouse kidney transplantation, STR: Surgical time on recipient, STD: Surgical time on donor, TIT: Total ischemia time. (B) Ischemia time in mouse kidney transplantation. TIT: Total ischemia time, CIT: Cold ischemia time, WIT: Warm ischemia time.

A

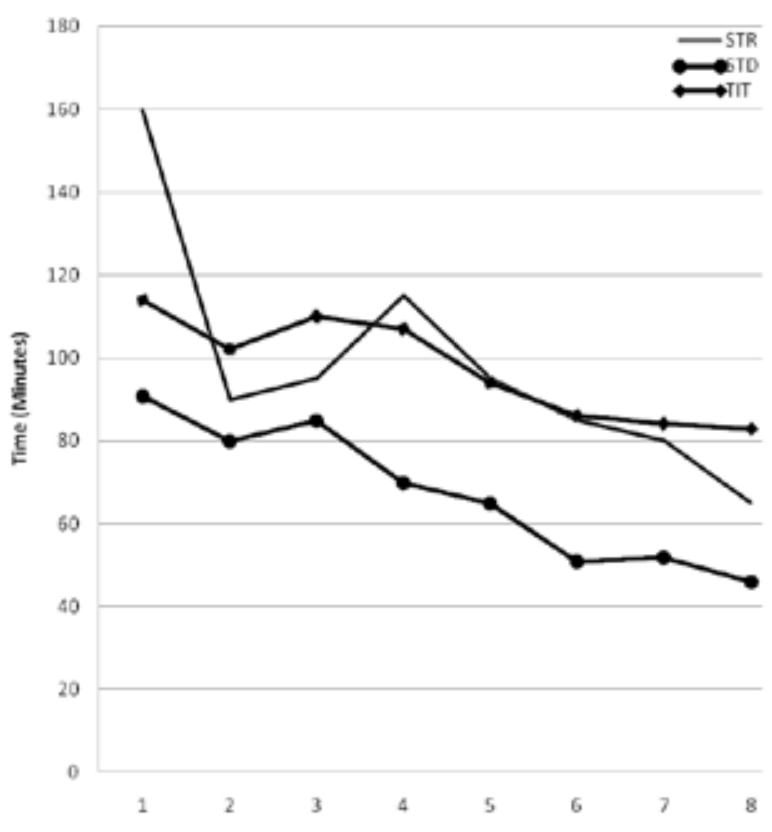

B




Table 3 - Ischemia times in different groups.

\begin{tabular}{|c|c|c|c|c|c|c|}
\hline & TIT & $p$ & WIT & $P$ & CIT & $p$ \\
\hline Thrombosis & $97.50 \pm 17.77$ & - & $37.50 \pm 10.34$ & - & $60.42 \pm 19.12$ & - \\
\hline Kidney failure & $85.77 \pm 17.67$ & \# 0.057 & $34.62 \pm 7.217$ & $\# 0.397$ & $51.54 \pm 9.12$ & \# 0.116 \\
\hline 30-day survival & $82.50 \pm 8.80$ & $\begin{array}{c}* 0.052 \\
* * 0.657\end{array}$ & $34.17 \pm 5.85$ & $\begin{array}{c}{ }^{*} 0.433 \\
* * 0.914\end{array}$ & $46.67 \pm 6.06$ & $\begin{array}{l}{ }^{*} 0.054 \\
* * 0.476\end{array}$ \\
\hline
\end{tabular}

No significant differences were found among mice of thrombosis, kidney failure and 30-day survival on various ischemia times.

\#: Compared with group of thrombosis. *: Compared with group of thrombosis.

**: Compared with group of kidney failure.

TIT (Total ischemia time): Time duration without perfusion. WIT (Warm ischemia time): Time duration on vessel anastomosis (out of cold saline preserve). CIT (Cold ischemia time): Time duration preserved in cold saline.

As a 10-year experienced cardiothoracic surgeon, microsurgery on mouse was still a challenge work which amalgamating manual precision and technical experience in equal measure. For the author, it took nearly 2 months and 30 mice surgery to get the first long-term survival kidney transplantation mouse. Practice is one of the basic factors for surgical results in surgical training. In this study, practice could significantly decrease the incidence of bleeding and increase surgical shortterm survival rate. The condition was similar in clinical surgeon training. However, no statistical difference was found of practice on long-term survival rate and complications including thrombosis and kidney failure. This may due to surgical techniques used in the suture of artery. When the suture method was switched from continuous to interrupted sutures on artery anastomosis, the incidence of thrombosis and kidney failure decreased significantly, and the long-term survival rate improved significantly. This indicated that in microsurgery procedure, besides practice, selection of the right surgical technique was equally important for the surgical results.

Successful mouse kidney transplantation is a surgical masterpiece. A variety of complications can lead to death or surgical failure. In our operation, surgical complications included bleeding, thrombosis, kidney failure, hydronephrosis, urine leakage, vena cava complication (stenosis and distortion) and breakdown of the skin suture. Bleeding is the most frequent cause of early postoperative mortality early in the learning curve. Mice have low tolerance to blood loss because of their relatively small blood volume. For a $20 \mathrm{~g}$ mouse, $0.14 \mathrm{~mL}$ blood loss (10\% total blood volume) can cause early postoperative mortality. In addition to improvement of microsurgical technique, Surgicel $^{\circledR}$ is an effective material that prevents bleeding. Thrombosis of the arterial anastomosis is another significant problem in mice kidney transplantation, and it was the most common technical complication after bleeding in this project. Similar to the experience of other researchers (6), we also found that mismatch of the recipient aortotomy and donor aorta was a factor leading to arterial thrombosis. Researchers should consider the factors that lead to complications when developing standard operating procedures.

Ureter implantation technique was used to reconstruct the ureter-bladder connection. This technique could significantly reduce ureter attachment time and the incidence of urine leakage (5). A common complication in this study was hydronephrosis, with a total of five cases in survived mice. Among the five cases, four cases appeared in Month 5 which was part of the reason resulting in low 30-day survival rate with high 3-day 
survival rate. The obstruction was always located in the junction of the ureter-bladder connection. This complication occurred when continuous suture was used. We expected that this suture method would prevent urine leakage. However, there was no urine leak in mice using three interrupted stitches around the junction of ureter-bladder connection, and also there were no hydronephrosis cases when this sewing method was used.

Ischemia time is one of the important factors impacting transplantation results (8). Ischemia itself can cause severe damage because of the potential for a build-up of metabolic wastes. Reperfusion after a period of ischemia can actually be more damage than the ischemia itself. Ischemia-reperfusion results in vascular edema and leukocytes building up in small capillaries and obstructing them which accelerated the process of thrombosis and renal failure after transplantation. In this study, the average total ischemia time ranged from 65 to 135 minutes, and warm ischemia time from 20 to 55 minutes, but there were no significant difference among 30-day survival, thrombosis and kidney failure mice. This shows that within a certain range, ischemia time was not the key factor for successful kidney transplantation. The same result was found by Tian (9), who found that the kidney graft can be stored in a cold solution for more than 2.5 hours, and there was no significant difference in success rate compared with a kidney graft stored for less than 1 hour.

Kidney failure is one of the most common complications in our procedure. The transplanted kidney looked pink with the renal artery pulse observed before closing the abdomen incision, and even on day 10 of post-transplant. However, the mice died 2 or 3 days after the other native kidney was took out. In this study, no relationship was found between kidney failure and ischemia time. When interrupted artery anastomosis was used, complication of kidney failure decreased significantly. Making the artery anastomosis with continuous suture would be more time-efficient and could decrease bleeding. But it may form a stenosis ring around the anastomosis site and limit the relaxation of the vessel. This is a significant problem for microvessels. The caliber of the mouse aorta is extremely small $(<0.4 \mathrm{~mm})$, so a $0.1 \mathrm{~mm}$ diameter change in a $0.4 \mathrm{~mm}$ diameter vessel can result in a nearly $60 \%$ cross-sectional area decrease. But in surgery, the $0.1 \mathrm{~mm}$ is a very small change. After bilateral nephrectomy, blood flow can't increase proportionately because of the constriction of the continuous suture. In addition, the constriction ring could lead to formation of micro-thrombosis. As hypothesized, the incidence of kidney failure decreased significantly after adoption of interrupted suture. For the anastomosis of artery in small animal like mouse, the experience of this study indicated that interrupted suture method would be a better choice compared with continuous suture.

In summary, successful mouse kidney transplantation is a challenging microsurgical procedure. In our experience, practice is the base of microsurgery success, and surgical techniques choice can change the surgical results significantly. There are some factors such as practice and the use of interrupted arterial sutures that are essential to prevent bleeding, thrombosis and kidney failure, and to increase the long-term survival rate. In this study, however, ischemic time was not a factor in determining the success of kidney transplantation in mice.

\section{CONFLICT OF INTEREST}

None declared.

\section{REFERENCES}

1. Mannon RB, Doyle C, Griffiths R, Bustos M, Platt JL, Coffman TM.: Itered intragraft immune responses and improved renal function in MHC class II-deficient mouse kidney allografts. Transplantation. 2000; 69: 2137-43.

2. Wang C, Cordoba S, Hu M, Bertolino P, Bowen DG, Sharland AF, et al.: Spontaneous acceptance of mouse kidney allografts is associated with increased Foxp3 expression and differences in the B and T cell compartments. Transpl Immunol. 2011; 24: 149-56.

3. Skoskiewicz M, Chase C, Winn HJ, Russell PS: Kidney transplants between mice of graded immunogenetic diversity. Transplant Proc. 1973; 5: 721-5.

4. Zhang Z, Schlachta C, Duff J, Stiller C, Grant D, Zhong R: Improved techniques for kidney transplantation in mice. Microsurgery. 1995; 16: 103-9. 
5. Han WR, Murray-Segal LJ, Mottram PL: Modified technique for kidney transplantation in mice. Microsurgery. 1999; 19: 272-4.

6. Martins PN: Learning curve, surgical results and operative complications for kidney transplantation in mice. Microsurgery. 2006; 26: 590-3.

7. Ge F, Gong W: Strategies for successfully establishing a kidney transplant in a mouse model. Exp Clin Transplant. 2011; 9: 287-94.
8. Gok MA, Shenton BK, Pelsers M, Whitwood A, Mantle D, Cornell $C$, et al:: Ischemia-reperfusion injury in cadaveric nonheart beating, cadaveric heart beating and live donor renal transplants. J Urol. 2006; 175: 641-7.

9. Tian Y, Chen J, Gaspert A, Segerer S, Clavien PA, Wüthrich $\mathrm{RP}$, et al.: Kidney transplantation in mice using left and right kidney grafts. J Surg Res. 2010; 163: e91-7.

Correspondence address

Dr. Ling-Jin Huang

Department of Cardiothoracic Surgery Xiangya Hospital

Central South University, Changsha, Hunan, 410008, PRC Fax: +86 $7318432-7247$

E-mail: drhuanglj@yahoo.com.cn

\section{EDITORIAL COMMENT}

The basic research is so important as clinical trials, but few studies have been published about some topics.

Kidney transplant in mouse model is quite useful and valuable for studying transplant immunology and surgery techniques. However, the technical difficulty and high mortality are a huge obstacle in its widespread use.

Animal models are commonly used to study the pathogenesis of acute and chronic diseases. The use of mouse as models are much valuable in researches because the development of gene knockout and transgenic animals can lead to better control of the studies.

In this study the authors evidenced which factors affected kidney transplants in a mouse model. Although in recent review of the literature
(1), Ge and Gong, found that the warm and cold ischemia time should be less than 35 five minutes and 2.5 hours, this study evidenced that ischemic time was not a factor in determining the successful of kidney transplantation.

The information obtained by the authors in this study have contributed to improve what Zheng et al. have had describe (2), no matter the mouse kidney transplants successful have still a challenge.

The renal failure treatment by transplants have had excellent outcomes nowadays, although these outcomes, physicians who have been working in this Field keep on their researches up to date as the way to improve the outcomes of transplants, so the data showed in this article will help them to improve their basic researches.

\section{REFERENCES}

1. Ge F, Gong W: Strategies for successfully establishing a kidney transplant in a mouse model. Exp Clin Transplant. 2011; 9: 287-94.

2. Zhang Z, Schlachta C, Duff J, Stiller C, Grant D, Zhong R: Improved techniques for kidney transplantation in mice. Microsurgery. 1995; 16: 103-9.

\section{Dr. Luiz Carlos Maciel Hospital Ipiranga} Av. Nazaré, 28 - Ipiranga São Paulo, SP, 04262-000, Brazil

E-mail: luizmaciel@uol.com.br 


\section{EDITORIAL COMMENT}

Clinical kidney transplantation has become the preferred method of renal replacement therapy worldwide. Testing new pharmacological and biological agents, and identifying new methods to diagnose and treat rejection depends on preclinical investigation. The use of large animal models such as the monkey, dog, cat, pig, etc. in transplant research has become problematic due to high cost, the need for specialized animal care, longer gestation and life spans, and cultural sensitivities regarding certain species.

The rat is of sufficient size (300-400 grams) to permit solid organ transplant experiments using microsurgical techniques, but has been less well characterized than the mouse in molecular biology. However, the adult mouse (30-50 grams) is $5-10$ times smaller than the rat, more difficult to perform microsurgery, and ultimately more prone to technical failures (1).

The mouse model has been used for research since there are more than 1300 mutant mice with which to study the biology of diseases, particularly the complexity of the immune system (2). Indeed, the mouse has many inherent advantages: low cost, no specialized animal care, short gestation, short life spans, and no culture sensitivities. The mouse model of a kidney transplant was first

\section{REFERENCES}

1. Martins PN: Technique of kidney transplantation in mice with anti-reflux urinary reconstruction. Int Braz J Urol. 2006; 32: 713-8; discussion 719-20.

2. Erickson RP: Mouse models of human genetic disease: which mouse is more like a man? Bioessays. 1996; 18: 993-8.

3. Skoskiewicz M, Chase C, Winn HJ, Russell PS: Kidney transplants between mice of graded immunogenetic diversity. Transplant Proc. 1973; 5: 721-5.

4. Zhang Z, Schlachta C, Duff J, Stiller C, Grant D, Zhong R: Improved techniques for kidney transplantation in mice. Microsurgery. 1995; 16: 103-9.

5. Wang M, Bai J, Baumann M, Heemann U: New model for simultaneous heart and kidney transplantation in mice. Microsurgery. 2003; 23: 164-8. reported in 1973 by Skoskiewicz and associates (3). However, the demanding techniques and mortality rates have impeded widespread use of this model.

The learning curve in kidney transplantation in mice is substantially longer than in rats and the mortality rate is as high as 50\%, even in the hands of experienced microsurgeons and long-term survival rates are sparse. Most animals died due to surgical related complications.

The end-to-side procedure for vascular reconstruction is widely used for establishing a mouse model of kidney transplant. Continuous sutures are preferred for vascular anastomosis with high success (4-8).

The authors describe a change in the surgical technique carrying out the arterial anastomosis with an interrupted suture which factor was responsible for the highest survival index at the thirtieth day, probably due to a lesser incidence of kidney failure without however altering the time of warm isquemia. This technique must be reproduced in future studies aiming to diminish technical failures consequently high levels of animal mortality, with the objective to stimulate a wider use of such kidney transplants as models for a long term follow-up.
6. Bickerstaff A, Pelletier R, Wang JJ, Nadasdy G, DiPaola N, Orosz $\mathrm{C}$, et al.: An experimental model of acute humoral rejection of renal allografts associated with concomitant cellular rejection. Am J Pathol. 2008; 173: 347-57.

7. Russell PS, Chase CM, Colvin RB, Plate JM: Kidney transplants in mice. An analysis of the immune status of mice bearing long-term, $\mathrm{H}-2$ incompatible transplants. J Exp Med. 1978; 147: 1449-68.

8. Ge F, Gong W: Strategies for successfully establishing a kidney transplant in a mouse model. Exp Clin Transplant. 2011; 9: 287-94.
Dr. Fernando Meyer Federal University of Parana Rua XV de Novembro, 1299, Centro Curitiba, PR, 80.060-000, Brazil Fax: +55 41 3015-0303 E-mail:fmeyer@onda.com.br 